\title{
Efficiency of Hybrid Algorithms for Estimating the Shear Strength of Deep Reinforced Concrete Beams
}

\author{
Mohammad Sadegh Barkhordari ${ }^{1}$, De-Cheng Feng ${ }^{2}$, Mohsen Tehranizadeh ${ }^{\text {* }}$ \\ ${ }^{1}$ Department of Civil \& Environmental Engineering, Amirkabir University of Technology (Tehran Polytechnic), Tehran 1591634311, Iran \\ ${ }^{2}$ Key Laboratory of Concrete and Prestressed Concrete Structures of the Ministry of Education, Southeast University, Nanjing 211189 , \\ China \\ *Corresponding author, e-mail: tehranizadeh@aut.ac.ir
}

Received: 28 September 2021, Accepted: 07 January 2022, Published online: 12 January 2022

\begin{abstract}
Earthquakes occurred in recent years have highlighted the need to examine the strength of reinforced concrete (RC) members. $\mathrm{RC}$ beams are one of the elements of reinforced concrete structures. Due to the dramatic increase in the population and the number of medium/high-rise buildings, in recent years, the beams of buildings have been mainly designed and executed in the type of deep beams. In this study, the artificial neural network (ANN) with optimization algorithms, including particle swarm optimization (PSO), Archimedes optimization algorithm (AOA), and sparrow search algorithm (SSA), are used to determine the shear strength of reinforced concrete deep (RCD) beams. 271 samples from experimental tests are employed to develop algorithms. The results of this study, design codes equations, and previous research are compared. Comparison between the results shows that the PSO-ANN algorithm is more accurate than previous methods. Finally, SHApley Additive exPlanations (SHAP) method is utilized to explain the predictions. SHAP reveals that the beam span and the ratio of the beam span to beam depth have the highest impact in predicting shear strength.
\end{abstract} Keywords

deep reinforced concrete beams, hybrid algorithms, artificial neural networks, shear strength, machine learning

\section{Introduction}

Over the last few years, artificial intelligence has gotten a lot of attention in the field of civil engineering. Regarding structural and earthquake engineering, research on machine learning can be divided into three areas including structural health monitoring $[1,2]$, performance assessment of buildings [3-7], and prediction models of the mechanical behavior [8-12]. Estimating the response and predicting the behavior of structural members is one of the applications of machine learning techniques in civil engineering. Buildings are composed of various structural members such as beams, columns, slabs, and shear walls. One of the most important members of various structures is deep beams. Reinforced concrete deep (RCD) beams have various applications and are used in various structures such as pile caps, high-rise buildings, folded plate structures, among others. The shear failure mode is the most common failure mode in deep beams, and it has the potential to have severe results that jeopardize building safety. The flexure-shear interaction, concrete strength, the composite behavior of rebar and concrete, yield strength of reinforcement, size effect, reinforcement ratio, the ratio of effective depth, and other parameters influence the shear strength and shear behavior of deep beams. Recent research on the shear strength of $\mathrm{RC}$ beams resulted in the presentation of analytical and numerical methods such as the strut-and-tie model [13, 14], mechanism analysis, and the bound theorem of plasticity theory [15-17]. Researchers developed equations for calculating of the RCD beams' shear strength $[18,19]$. However, when the values obtained with these equations are compared to the results of experimental tests, at best, the values obtained for shear strength are conservative. On the other hand, machine learning techniques also were used for predicting the shear strength of the RCD beams. For instance, Feng et al. [9] used ensemble methods to estimate the RCD beams' shear capacity. However, there is no clear evidence that ensemble methods will perform better than others since some ensemble methods have a parallel structure and some have a series structure and the type of their structure affects their efficiency. Using gradient boosting regression, $\mathrm{Fu}$ and Feng [20] built a machine learning based technique 
for estimating the residual shear strength of corroded RC beams. Al-Musawi et al. [21] used support vector regression with a firefly optimization algorithm for predicting the shear strength of the steel fiber-reinforced concrete beams. Sharafati et al. [22] suggested novel models for the shear strength prediction of RC slender beams based on a combination of adaptive neuro-fuzzy system and meta-heuristic optimization techniques. Zhang et al. [23] proposed a new hybrid model based on a support vector regression algorithm and a genetic algorithm to predict the shear strength of the RCD beams using a total of 217 test records. The coefficient of determination of their model was 0.95 in the testing phase. Chou et al. [24] employed a firefly colony algorithm and support vector regression for predicting the shear strength of the RCD beams using 214 test results. The coefficient of determination of their model for the testing set was 0.92. Shahbazian et al. [25] calibrated the weights and biases of an artificial neural network (ANN) for forecasting the shear strength of reinforced concrete beams using the Tabu search training algorithm and 248 experimental results. For testing data, their model had an $\mathrm{R}^{2}$ value of 0.94 . Nikoo et al. [26] used 140 samples for predicting the shear strength of fiber reinforced polymer concrete beams using the bat optimization algorithm based ANN model. Nguyen et al. [27] employed the ANN to predict the shear strength of the RCD beams using a limited database containing 106 test results. Their results show that the ANN with conjugate gradient training algorithm had the best prediction performance with $\mathrm{R}^{2}=0.98$.

There has been an increase in the research on the ANN for analyzing structural engineering problems in recent years. An algorithm is used to discover a set of weights that optimally maps inputs to outputs when training the ANNs. It is a difficult task since the error surface is non-convex, has local minima and flat patches, and is extremely multidimensional. To put it another way, the back-propagation algorithm, which is a widely used algorithm for training feedforward ANNs, is prone to getting stuck in the local minimum and also can be slow [28, 29]. An approach to addressing these problems is to use metaheuristic optimization algorithms such as the particle swarm optimization (PSO), which are inspired by certain physical or chemical processes.

The objectives of this work are (1) developing hybrid technique based on the PSO, Archimedes optimization algorithm (AOA), sparrow search algorithm (SSA), and the ANN for improving the prediction of the deep beams shear strength, (2) comparing classic the metaheuristic optimization algorithm (PSO) with recently developed metaheuristic optimization algorithms (AOA and SSA), and (3) comparing the result of the best hybrid model with previous research. The remainder of this work is organized as follows. In Section 2, the experimental data are explained. In Section 3, hybrid algorithms are described. The results are reported in Section 4. Finally, in Section 5, a summary and the study's conclusions are presented.

\section{Data gathering}

To develop the hybrid models, a total of 271 experimental data of the RCD beams, which is collected from the literature by Feng et al. [9], is used. The database also includes specimens without web reinforcements. Table 1 shows the input variables with their range, mean, and standard deviation. Shear strength is set as output system. In Table 1, $a$ is the distance of the applied load from the support, $\rho$ is longitudinal reinforcement, $f_{y}$ is yield strength, $b$ is beam width (Fig. 1), $f_{c}$ is concrete compressive strength, $h$ is beam height, $l_{0}$ is beam span, $h_{0}$ is depth to the centroid of rebars, $S_{v / h}$ is bar spacing in the vertical and horizontal direction, $\rho_{v, h}$ is web reinforcement ratio in the vertical and horizontal direction, and $f_{y, v / h}$ is web yield strength in the vertical and horizontal direction.

\section{Model development}

The artificial intelligence approaches are capable of capturing the complex nonlinear relationship that exists between

Table 1 Describing the input parameters

\begin{tabular}{|c|c|c|c|c|c|c|c|c|c|c|c|c|c|c|c|c|}
\hline & $f_{c}$ & $b$ & $h$ & $a$ & $h_{0}$ & $l_{0}$ & $a_{/} h_{0}$ & $l_{0 / h}$ & $s_{v}$ & $f_{y, v}$ & $\mathrm{pv}$ & $s_{h}$ & $f_{y, h}$ & $\rho_{h}$ & $f_{y}$ & $\rho$ \\
\hline mean & 30.89 & 122.81 & 523.48 & 467.73 & 469.01 & 1484.45 & 1.06 & 2.93 & 154.68 & 292.92 & 0.35 & 67.82 & 213.70 & 0.30 & 361.76 & 1.62 \\
\hline std & 14.83 & 44.24 & 147.67 & 238.31 & 145.17 & 642.37 & 0.52 & 1.06 & 122.82 & 175.85 & 0.41 & 96.03 & 207.34 & 0.42 & 86.70 & 0.71 \\
\hline $\min$ & 12.26 & 76 & 254 & 125 & 216 & 500 & 0.22 & 0.91 & 0 & 0 & 0 & 0 & 0 & 0 & 210 & 0.12 \\
\hline $25 \%$ & 21 & 100 & 400 & 254 & 360 & 900 & 0.54 & 2 & 50.8 & 210 & 0.12 & 0 & 0 & 0 & 300 & 1.245 \\
\hline $50 \%$ & 23.93 & 102 & 500 & 425 & 462.96 & 1500 & 1.06 & 3.14 & 152 & 375.2 & 0.28 & 69.9 & 246.8 & 0.21 & 410 & 1.56 \\
\hline $75 \%$ & 44.23 & 130 & 560 & 610 & 500 & 2000 & 1.5 & 3.57 & 209.55 & 437.4 & 0.48 & 101.25 & 437.4 & 0.45 & 431 & 1.94 \\
\hline $\max$ & 73.6 & 305 & 915 & 1290 & 844 & 4065 & 2.7 & 5 & 457.5 & 586 & 2.45 & 801 & 586 & 2.45 & 504.8 & 4.08 \\
\hline
\end{tabular}




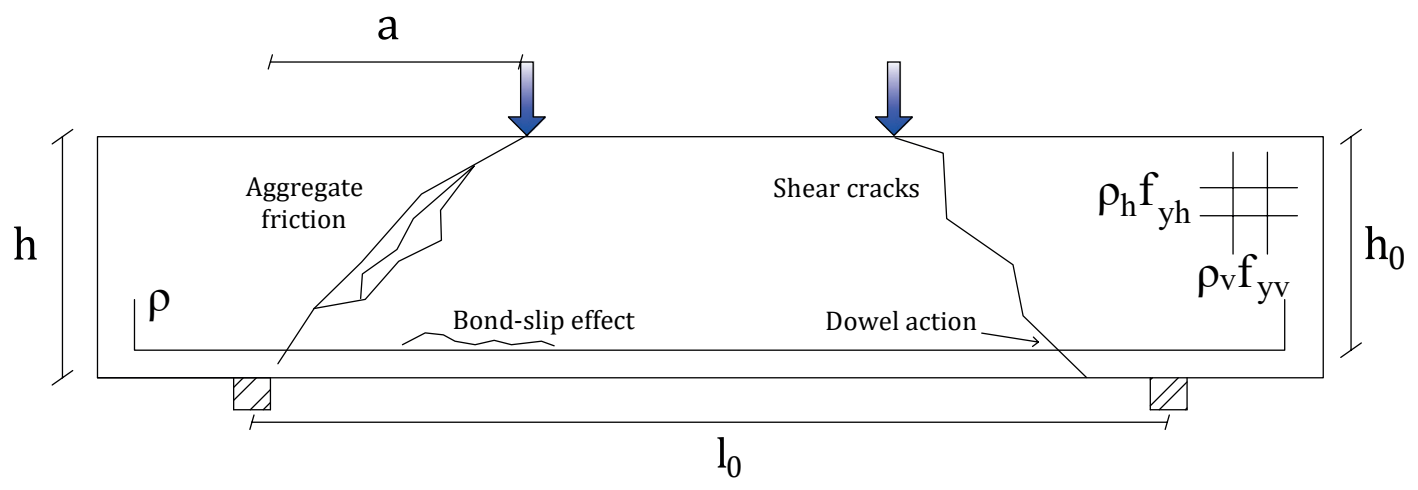

Fig. 1 Reinforced concrete deep beams

the shear strength of the RCD beams [9, 30-32]. For example, the ANN is used to solve civil engineering problems because it offers several advantages, including quick calculation and strong applicability. Despite the fact that some researchers tried to improve its performance by combining it with other methods, it suffers from limitations due to the tuning of its internal parameters. In this study, in order to establish a framework for the prediction of the shear strength of RCD beams, the ANN model is hybridized with the PSO, AOA, and SSA algorithms. The goal of merging these metaheuristic algorithms is to conduct autonomous detection of the best parameter settings (bias and weights) for the ANN. Because the models in the training phase are taught by knowing targets, the training phase cannot provide an acceptable assessment. As a result, the testing stage is critical in determining the model's efficiency because it introduces previously unseen target data, allowing the model's performance to be demonstrated. For training and testing datasets, different percentage values were proposed. Swingler [33], on the other hand, claims that a combination of $80 \%$ and $20 \%$ for network training and network testing produces the best model development and model evaluations. As a result, here, $80 \%$ of the whole data is utilized for training of the model and $20 \%$ of the total data is utilized for model evaluation. We also scales the inputs to be between -1 and 1. According to Hornik et al. [34], one hidden layer neural network can solve most non-linear functions. Hence, ANNs with only one hidden layer are considered in this study.

Particle swarm optimization is an optimization technique that uses a population-based approach. Improved PSO is widely employed [35], and it is the source of inspiration for a new field of research known as swarm intelligence. Particle swarm optimization has been used in a variety of settings. This algorithm searches for the best solution using particles whose paths are changed by both a deterministic and probabilistic component. The position vector $\boldsymbol{x}_{i}$ and the velocity vector $\boldsymbol{v}_{i}$ define a particle $i$. At iteration $t$, the particle swarm optimization employs both the global best $\left(\right.$ BBest $\left._{i}^{t}\right)$ and the individual best $\left(x\right.$ Best $\left._{i}^{t}\right)$ to determine each particle position (Eq. (1)). One of the motivations for adopting individual best is to generate diverse efficient solutions; however, this variety can be mimicked using some randomization.

$$
\begin{aligned}
& v_{i}^{t+1}=\omega v_{i}^{t}+c_{1} r_{1}\left(x \text { Best }_{i}^{t}-x_{i}^{t}\right)+c_{2} r_{2}\left(\text { gBest } t_{i}^{t}-x_{i}^{t}\right) \\
& x_{i}^{t+1}=x_{i}^{t}+v_{i}^{t} . t
\end{aligned}
$$

In Eq. (1), the parameters $\omega, c_{1}, c_{2}, r_{1}$ and $r_{2}$ are inertia weight, two positive constants, and two random parameters within $[0,1]$, respectively. The optimal static parameters are $\omega=0.72984$ and $c_{1}=c_{2}=2.05, c_{1}+c_{2}>4$ to create a standard PSO as suggested by [36]. The particle's movement in the solution space is depicted schematically in Fig. 2.

A neural network is a structure made up of numerous nodes connected by links. ANNs can learn to establish the relationship between the system's input and output by learning from the predefined training patterns. Each neuron in the network receives a weighted inputs. The signals are then processed by a particular activation function

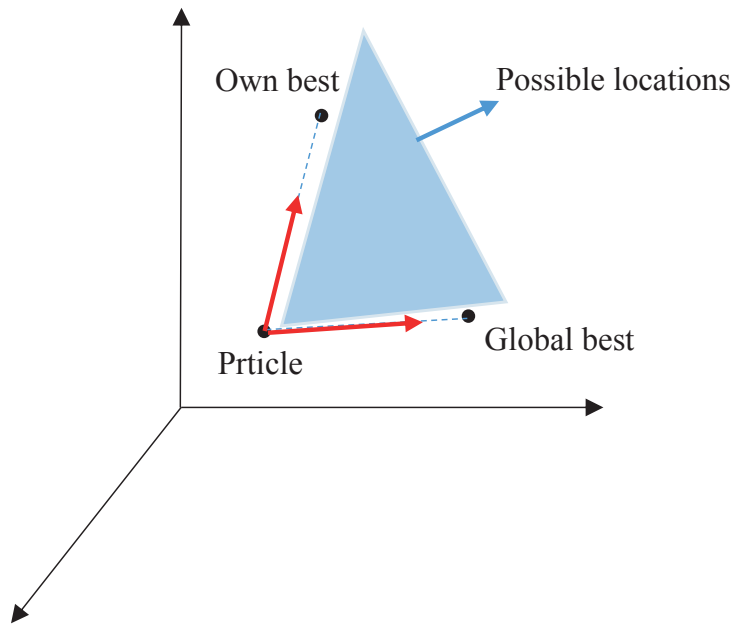

Fig. 2 The particle's movement in the solution space in PSO 


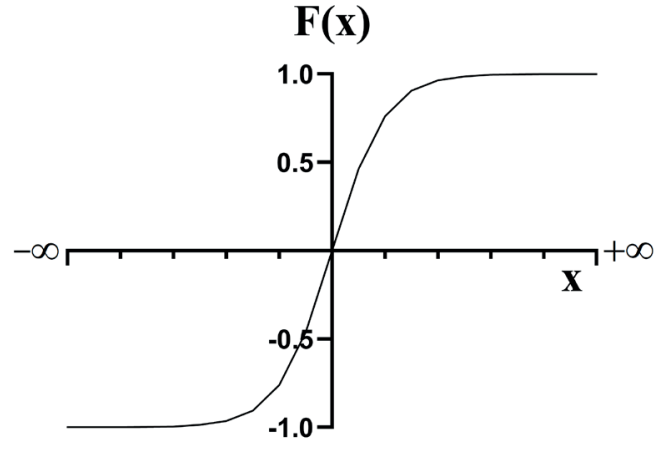

Fig. 3 Hyperbolic tangent sigmoid transfer function

to provide a meaningful output. The hyperbolic tangent sigmoid transfer function (Fig. 3) is utilized as an activation function (AF). The function creates outputs between 1 and -1 . The bias and weights of the ANNs with different number of neurons are determined using the PSO method.

As previously said, the goal of this research is to propose a novel intelligent methodology for high-accuracy shear strength prediction based on PSO and ANN. To do this, the PSO algorithm will conduct a global iterative process for the ANNs' weights and bias. The search results would then be the input of the ANNs for learning. The determination coefficient is computed as Eq. (2) and is utilized as a fitness function for assessing the efficacy of the trained ANNs.

$$
R^{2}=1-\frac{\sum_{i=1}^{n}\left(y_{i}-\bar{y}_{i}\right)^{2}}{\sum_{i=1}^{n}\left(y_{i}-\bar{y}_{i}\right)^{2}},
$$

where $n$ is the total of observations, $y_{i}$ is the measured values, $\hat{y}_{i}$ is the predicted value, and $\bar{y}_{i}$ the mean of measured values. The coefficient of determination ought to be the smallest for the optimal model. To identify the best values, the searching process can be performed several times (Fig. 4).

The Archimedes optimization algorithm (AOA) [37] is another population-based technique in which the members of a population are immersed particles. AOA is founded on Archimedes' principle, which is a physical rule. The initial set of particles (possible solutions) with randomly selected volumes $\left(\right.$ vol $\left._{i}\right)$, densities $\left(\right.$ den $\left._{i}\right)$, and accelerations $\left(a c c_{i}\right)$ is used by AOA to start the search strategy. Each object is also assigned to its arbitrary position in the fluid at this point. AOA performs in iterations until the cutoff condition is met after assessing the fitness of the current solutions. Every iteration, AOA changes each object's density and volume. The object's acceleration is modified depending on the state of its contact with every other nearby object. The objects strive to find equilibrium after collision. The transmission operator $(T F)$ and density decreasing factor $\left(d^{t+1}\right)$ in AOA are used to handle this situation (Eq. (3) and Eq. (4)).

$$
\begin{aligned}
& T F=\exp \left(\frac{t-t_{\max }}{t_{\max }}\right), \\
& d^{t+1}=\exp \left(\frac{t_{\text {max }}-t}{t_{\max }}\right)-\left(\frac{t}{t_{\max }}\right),
\end{aligned}
$$

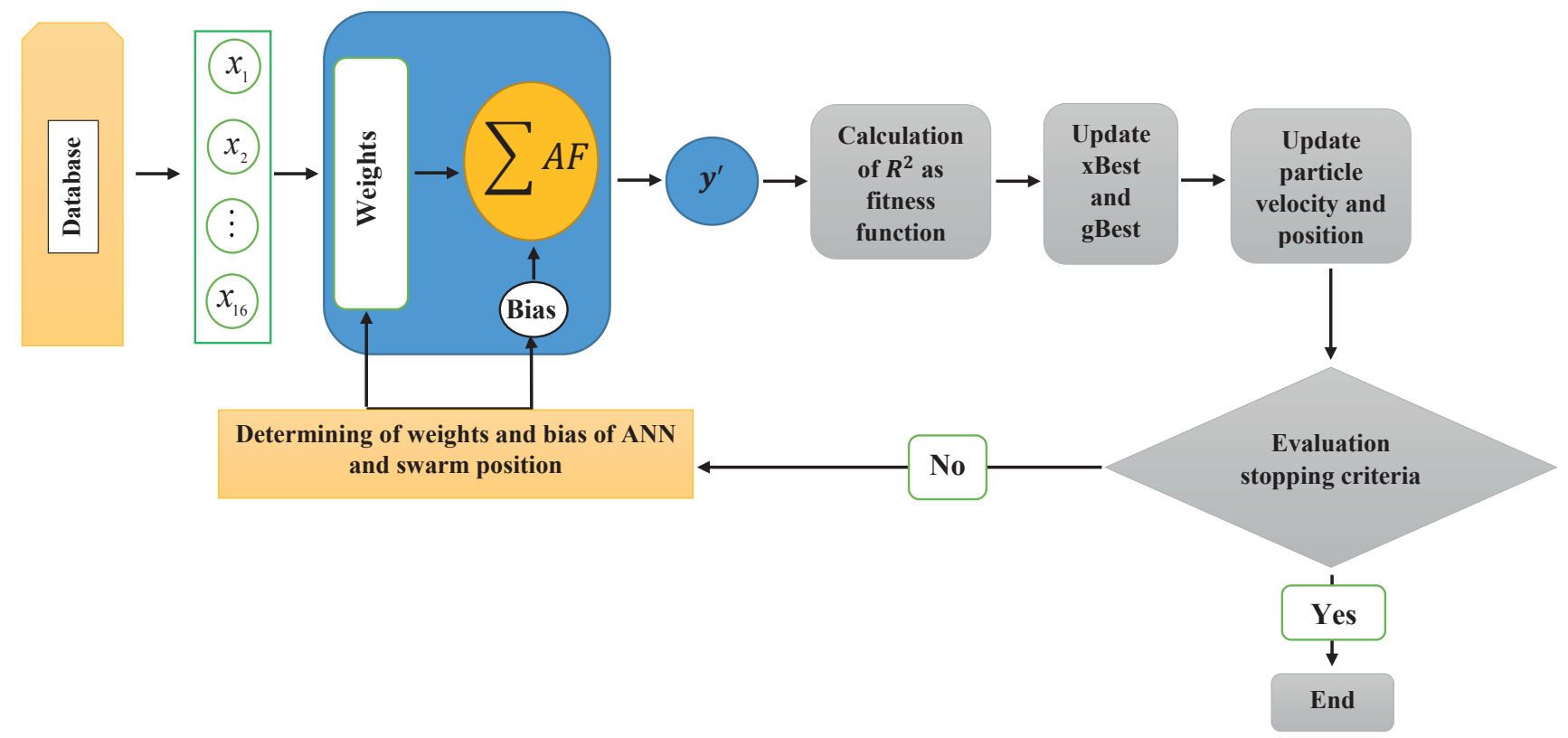

Fig. 4 Flowchart of the PSO-ANN algorithm 
where $t$ is iteration number and $t_{\max }$ is maximum iterations. If the TF value is less than 0.5 , it means that a collision has occurred between the particles. In this case, the particle acceleration is updated and then normalized using Eq. (5) and Eq. (6).

$$
\begin{aligned}
& a c c_{i}^{t+1}=\frac{\operatorname{den}_{m r}+v o l_{m r} \times a c c_{m r}}{d e n_{i}^{t+1} \times v o l_{i}^{t+1}}, \\
& a c c_{i-\text { norm }}^{t+1}=u \times \frac{a c c_{i}^{t+1}-\min \left(a c c_{i}\right)}{\max \left(a c c_{i}\right)-\min \left(a c c_{i}\right)}+1,
\end{aligned}
$$

where $u$ and 1 determine the range of normalization, $a c c_{i-n o r m}^{t+1}$ is normalized value, and the $m r$ index means select a random material. And the particle's position $\left(x_{i}^{t+1}\right)$ for the next iteration is updated using Eq. (7):

$x_{i}^{t+1}=x_{i}^{t}+2 \times a c c_{i-n o r m}^{t+1} \times d^{t+1} \times\left(x_{\text {rand }}-x_{i}^{t}\right)$,

where rand is uniformly distributed random number. If the TF is greater than 0.5 , there are no particles that collide. In this case, the particle acceleration is updated using Eq. (8), and the particles update their positions using Eq. (9).

$$
\begin{aligned}
& \operatorname{acc}_{i}^{t+1}=\frac{\operatorname{den}_{\text {best }}+\operatorname{vol}_{\text {best }} \times a c c_{\text {best }}}{\operatorname{den}_{i}^{t+1} \times \operatorname{vol}_{i}^{t+1}}, \\
& x_{i}^{t+1}=x_{\text {best }}^{t}+F \times 6 \times \text { rand } \times a c c_{i-\text { norm }}^{t+1} \times \\
& d^{t+1} \times\left(2 \times T F \times x_{\text {best }}-x_{i}^{t}\right), \\
& F=\left\{\begin{array}{ll}
+1 & \text { if }(2 \times \text { rand }-0.5) \leq 0.5 \\
-1 & \text { if }(2 \times \text { rand }-0.5)>0.5
\end{array},\right.
\end{aligned}
$$

where $a c c_{\text {best }}$ is the acceleration of the best particle, and $x_{\text {best }}$ is the position of the best particle. Here AOA algorithm is integrated with ANN to determine weights and bias. Fig. 5 shows the concept of AOA-ANN algorithm.

The sparrow search algorithm (SSA) [38] is a fairly new heuristic technique for swarm intelligence. When compared to standard heuristic search methods, it has a faster convergence time, a stronger optimization capability, and a wider range of application areas [38]. The producer/finders and the scrounger are two separate species of sparrows in a group. The SSA is proposed to handle the global optimization problem using bionics of interaction relations between producer and scrounger sparrows in the foraging procedure. The producers move in different directions

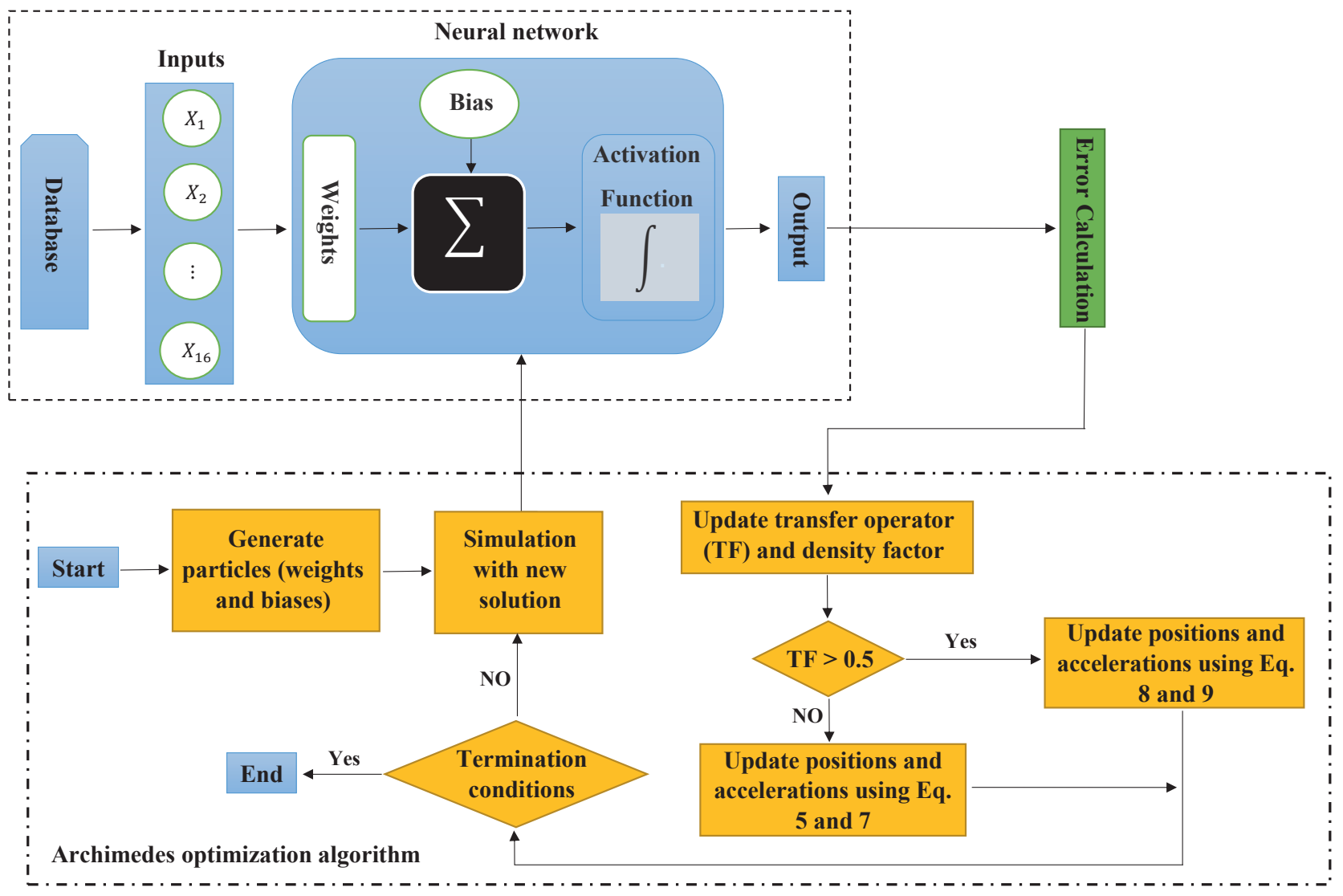

Fig. 5 Flowchart of the AOA-ANN algorithm 
to find food, while the scroungers rely on the producers to feed them. Moreover, research suggests that the birds flip back and forth between the producers and scrounging behavior. In SSA, a matrix represents the location of all sparrows (Eq. (10)), and the fitness values are allocated to the individuals. Producers, on average, have higher fitness values. The SSA uses Eq. (11) to update the positions of the producers.

$x_{i, j}=l b_{j}+\left(u b_{j}-l b_{l}\right) \times \operatorname{random}(1, \operatorname{dim})$,

$x_{i, j}^{t+1}=\left\{\begin{array}{ll}x_{i, j}^{t} \cdot \exp \left(\frac{-i}{\alpha \cdot \text { iter }_{\text {max }}}\right) & \text { if } R_{2}<S T \\ x_{i, j}^{t}+Q \cdot \boldsymbol{L} & \text { if } R_{2} \geq S T\end{array}\right.$,

where $x_{i j}$ signifies the $i^{\text {th }}$ point in the $j^{\text {th }}$ dimension of the solution space, $\alpha$ is a random number in $(0,1], l b_{j}$ and $u b_{j}$ indicate the $j^{\text {th }}$ dimension's lower and upper bounds, iter $r_{\max }$ is maximum number of iterations, random() is a dimensional vector randomly generates number in $[0,1]$, dim defines the searching space's dimension, $R_{2}$ is the alert number, $Q$ is a random number in $[0,1]$ that follows the rules of normal distribution, $\boldsymbol{L}$ is a $1 \times \operatorname{dim}$ matrix and its elements are 1 , and $S T$ is the safety level. $R_{2}<S T$ implies that the group environment is safe at this epoch, there are no enemies in the area, and the producers are free to seek food on a large scale. When $R_{2}$ is larger than or equal to $S T$, the individuals in the cluster have discovered a predator, and the producers will guide the follower/ scrounger to a safe spot. Equation (12) is used to update scroungers position.

$x_{I, J}^{t+1}=\left\{\begin{array}{l}Q \cdot \exp \left(\frac{x_{\text {worst }}^{t}-x_{i, j}^{t}}{i^{2}}\right) \text { if } i>n / 2 \\ x_{o}^{t+1}+\left|x_{i, j}^{t}-x_{o}^{t+1}\right| \cdot \boldsymbol{A}^{+} \cdot \boldsymbol{L} \\ \boldsymbol{A}^{+}=\boldsymbol{A}^{T}\left(\boldsymbol{A} \boldsymbol{A}^{T}\right)^{-1} \quad \text { otherwise }\end{array}\right.$,

where $x_{o}$ is the optimal position, $x_{\text {worst }}$ is the worst position, $\boldsymbol{A}$ is a $1 \times d$ matrix ( $d$ is the dimension of the problem) in which each element is allocated 1 or -1 at random, and $i>n / 2$ indicates that the sparrow group is aware of the danger. The SSA algorithm is also integrated with ANN to determine weights and bias. Fig. 6 shows the concept of SSA-ANN algorithm.

\section{Result and discussion}

The ANN has a single layer of neurons, as indicated above in terms of network topology. As a result, we must provide values for the number of neurons. The number of neurons must be optimized in order to develop accurate predictive models. To find the suitable values for the number of neurons in the hidden layer, a trial and error procedure

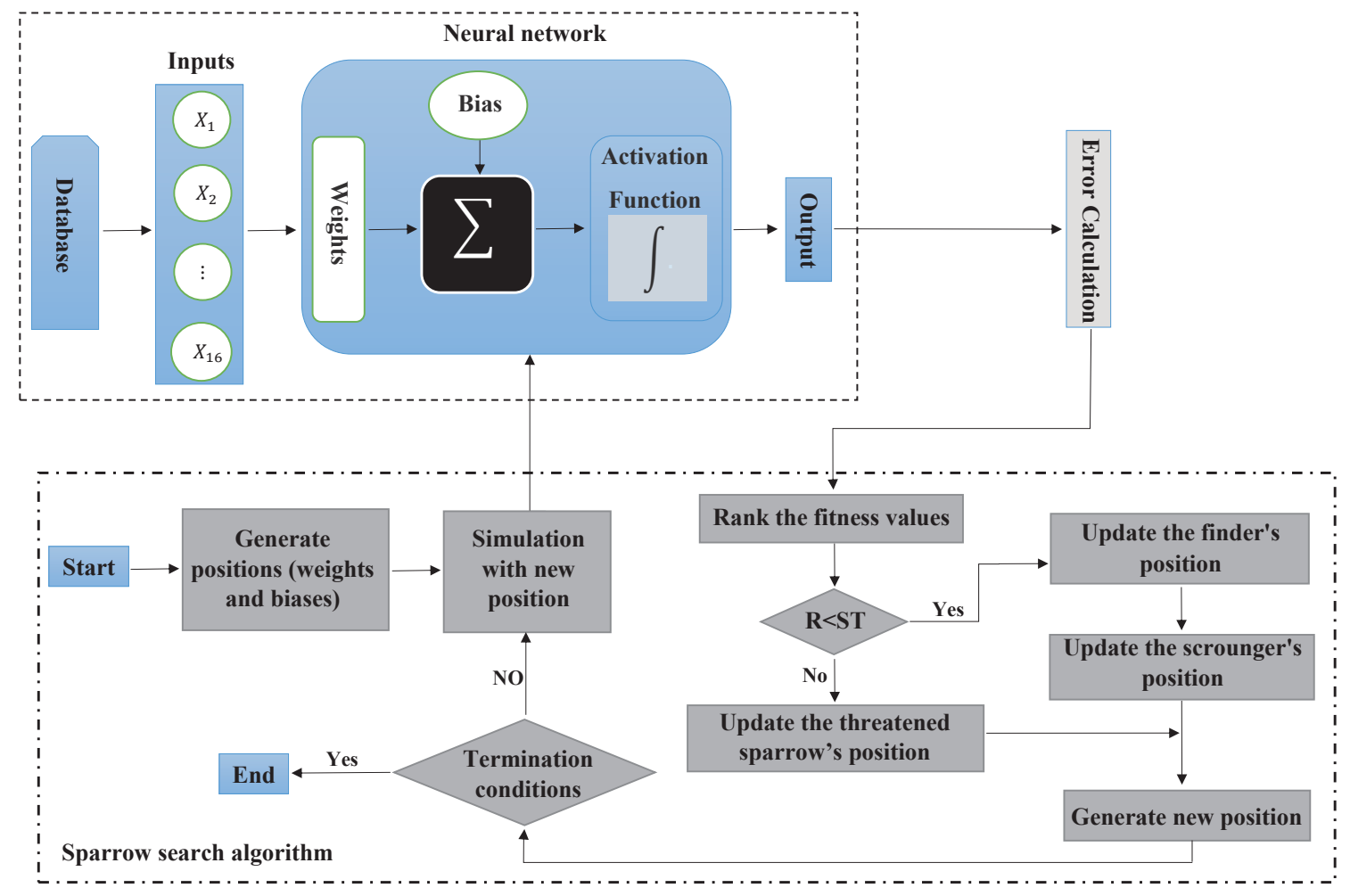

Fig. 6 Flowchart of the SSA-ANN algorithm 
was performed. There should also be a balance between computational cost and accuracy because a large number of neurons means an increase in the parameters that need to be adjusted [39]. In this study, 3-20 neurons were examined (18 ANNs with different number of neurons), and each hybrid model was run numerous times to determine the optimal performance. For these three methods, the population size and number of iterations were 200 and 1000, respectively. Fig. 7 shows the $R^{2}$ diagram for the hybrid algorithms. The maximum $R^{2}$ value for PSO-ANN, SSA-ANN, and AOA-ANN models is $0.955,0.955$, and

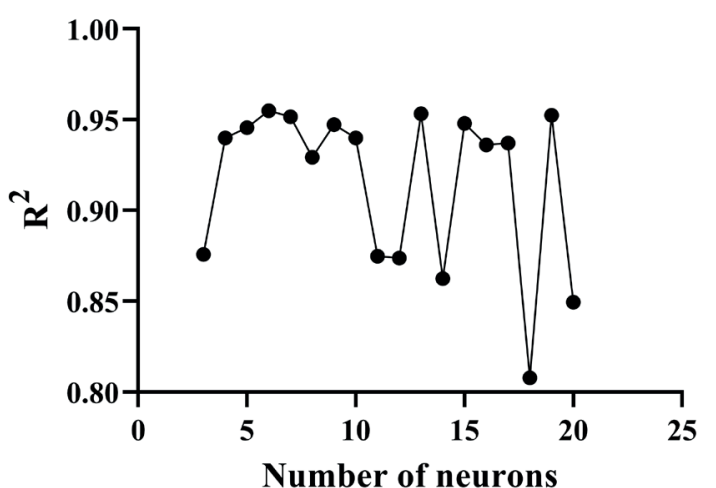

(a)

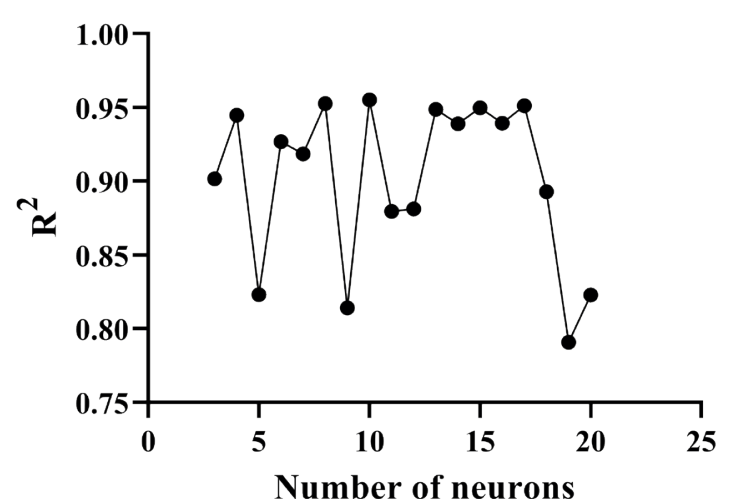

(b)

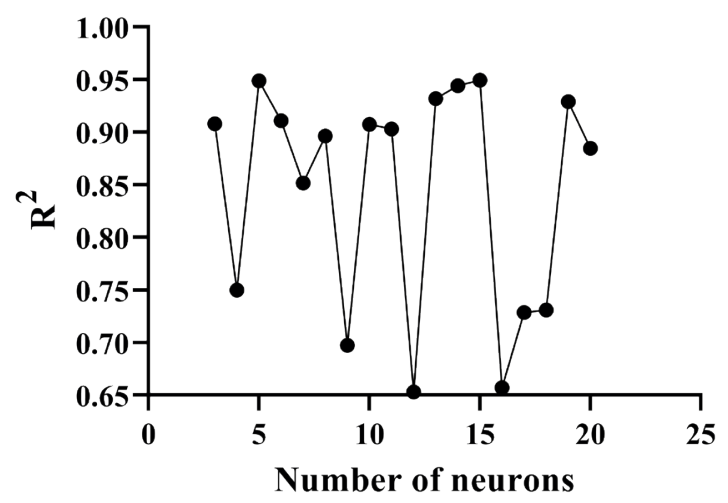

(c)

Fig. 7 Effect of neuron number on models performance; a) PSO-ANN algorithm, b) SSA-ANN algorithm, c) AOA_ANN algorithm
0.949, respectively. The accuracy of the PSO-ANN and SSA-ANN models is the same, but the first model is able to achieve this accuracy with a smaller number of neurons. For each algorithm (PSO-ANN, SSA-ANN, and AOAANN), the model that has the highest accuracy with the least number of neurons is selected as the best model. For example, a good performance is provided with 6 neurons for the PSO-ANN. Therefore, the model with 6 neurons is select as best PSO-ANN model. The Taylor diagram of the best models is shown in Fig. 8. Because the Taylor diagram is created using three statistical indicators (correlation coefficient, the root-mean-square (RMS) error, and the standard deviation), it eliminates the redundancy of the statistical metrics. Predicted values that fitted well with data, have a high correlation coefficient as well as low root-mean-square errors, will be closest to the $\mathrm{x}$-axis point labeled "observed". It is evident from Fig. 8 that for the PSO-ANN, the correlation coefficient is about 0.975 , the RMS error is less than 0.1, and the standard deviation is about 0.35. The PSO-ANN and SSA-ANN models generally agree better with observations. The PSOANN; however, almost has the same standard deviation as the observed field (indicated by the solid-red contour). Hence, The PSO-ANN algorithm is selected as the best model. Tables 2 to 4 present the relevant weight coefficients $\left(W_{i j}, W_{j k}^{\prime}\right)$ and biases $\left(B_{i}\right)$ of the PSO-ANN algorithm (Fig. 9). The performance of the PSO-ANN is tested through repeated 10 -fold cross-validation since distinct data splits can generate significantly different results. This includes basically repeating the cross-validation process more than once and reporting the mean result across all folds from all runs. This mean result, obtained using the

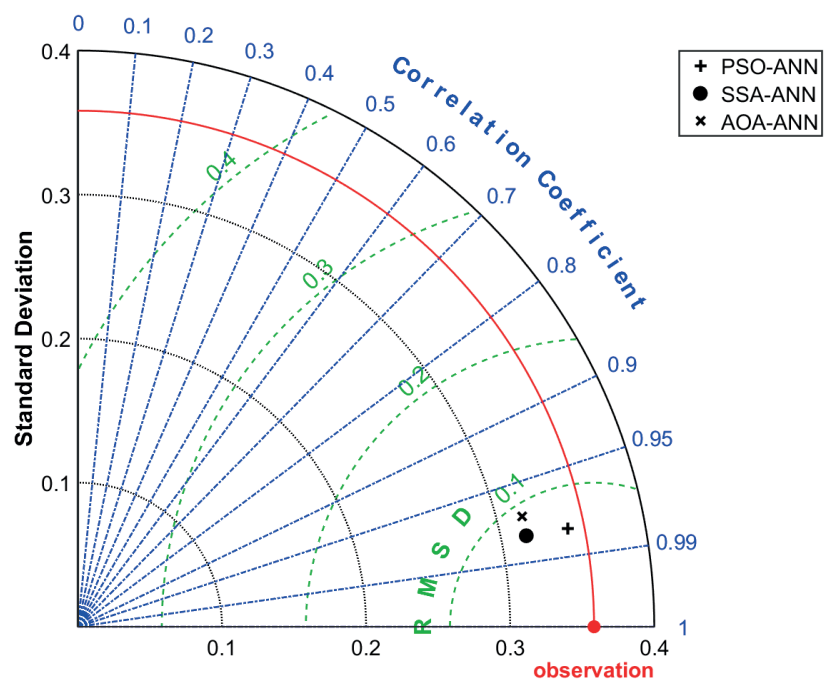

Fig. 8 Taylor diagram of the various models 
Table 2 The weight coefficients $\left(W_{i j}\right)$

\begin{tabular}{ccccccccccccccccccccc}
\hline & $\mathrm{I}=1$ & $i=2$ & $i=3$ & $i=4$ & $i=5$ & $i=6$ & $i=7$ & $i=8$ & $i=9$ & $i=10$ & $i=11$ & $i=12$ & $i=13$ & $i=14$ & $i=15$ & $i=16$ \\
\hline$j=1$ & 0.0437 & 1 & 0.1472 & -0.4769 & 0.1123 & -0.2138 & -0.4583 & -0.027 & 0.0139 & -0.4081 & 0.0287 & -0.1084 & 0.4532 & -0.4456 & 1 & 0.0143 \\
$j=2$ & 0.6201 & -0.4306 & 0.3785 & 0.9438 & -1 & 0.1401 & -0.541 & -0.9909 & 0.1206 & 0.3113 & 0.1136 & 0.0231 & -0.6996 & -0.6151 & 0.9575 & 0.062 \\
$j=3$ & 0.1846 & 0.547 & 0.1857 & -0.1585 & 0.1508 & -0.0835 & -0.715 & 0.3379 & -0.435 & 0.0682 & 0.1265 & -0.1736 & -0.2786 & -0.184 & 0.1319 & 0.8439 \\
$j=4$ & 0.1011 & -0.9427 & -0.3796 & -0.0902 & 0.0553 & -0.299 & -0.2223 & 0.1436 & -0.1132 & -0.2604 & 0.1998 & -0.2824 & -0.1015 & -0.3118 & -0.2774 & -0.2738 \\
$j=5$ & -0.6216 & 0.3233 & -0.1093 & -1 & 0.375 & -0.2179 & -0.22 & 0.7313 & 1 & -0.6285 & 0.151 & -0.3003 & 1 & 0.1944 & -0.2672 & -0.4275 \\
$j=6$ & 1 & 0.4237 & 0.1555 & -0.1223 & 0.6119 & -0.1914 & -0.8868 & -0.0445 & -0.0025 & 0.1012 & 0.3445 & -0.6935 & -0.0481 & 0.0843 & -0.4194 & 0.3897 \\
\hline
\end{tabular}

Table 3 The weight coefficients $\left(W_{i j}^{\prime}\right)$

\begin{tabular}{|c|c|c|c|c|c|c|}
\hline & $j=1$ & $j=2$ & $j=3$ & $j=4$ & $j=5$ & $j=6$ \\
\hline$k=1$ & 0.4751 & -0.0754 & 0.2935 & -0.8609 & 0.1331 & 0.5052 \\
\hline \multicolumn{7}{|c|}{ Table 4 Biases value } \\
\hline & $j=1$ & $j=2$ & $j=3$ & $j=4$ & $j=5$ & $j=6$ \\
\hline $\mathrm{B}_{1}$ & -0.9266 & 0.4358 & 0.0718 & 0.0725 & -0.3496 & 0.5536 \\
\hline $\mathrm{B}_{2}$ & \multicolumn{6}{|c|}{-0.0255} \\
\hline
\end{tabular}

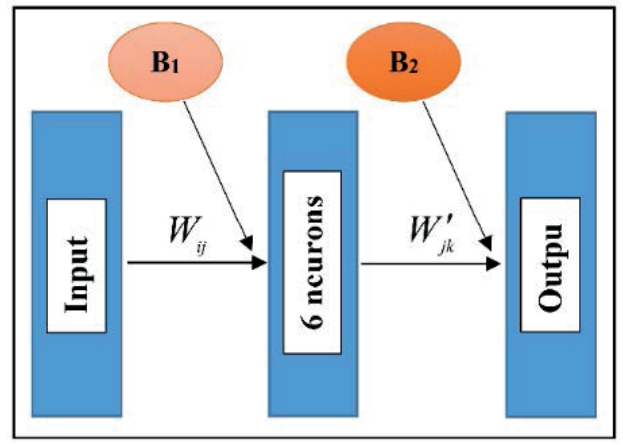

Fig. 9 Neural network configuration

standard error, is believed to be a more reliable representation of the genuine unknown underlying average model performance on the database [40, 41]. Fig. 10 shows the results of repeated 10 -fold cross-validation for the PSOANN model. The arithmetic mean and the median are represented by the green triangle and the orange line, respectively. The graph illustrates the average fluctuates slightly around 0.955 . Hybrid model outperforms the back-propagation of error algorithm or stochastic gradient descent algorithm. Optimization algorithms are a systematic search, but instead of random or completely exploring the space of possible solutions, it employs any existing knowledge to choose the next step in the searching, such as a set of weights with reduced error [42]. Regarding computational efficiency, optimization algorithms do not require calculating gradients for the weights [42]. In addition, optimization algorithms are less likely to become trapped in local minima [35].

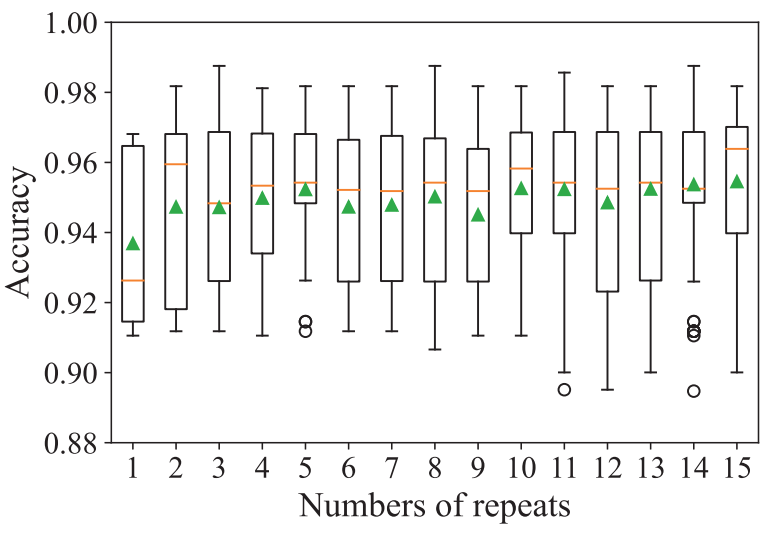

Fig. 10 Results of repeated 10-fold cross-validation for the PSO-ANN model

\subsection{Comparison with previous studies}

In order to evaluate the efficiency of the proposed PSOANN model, the results of the present study are compared with the results of recent research on predicting the RCD beams' shear strength using machine learning techniques. Feng et al [9] used ensemble learning techniques to train prediction models. They used random forest (RF), adoptive boosting (AdaBoost), gradient boosting regression tree, and eXtreme Gradient Boosting (XGBoost) to estimate the shear strength of deep beams. RF is a collection of many separate decision trees that work together to form an ensemble. A prediction is generated by each individual tree in the random forest. Since a lot of relatively low uncorrelated trees operate as a group, they surpass any of the individual models. AdaBoost is one of the boosting algorithms to be used in problem-solving. Adaboost makes it possible to merge numerous weak classifiers and create a single strong classifier. Both classification and regression problems can be solved with AdaBoost algorithms. AdaBoost works by giving more attention to cases that are difficult to categorize and less to those that are already well-classified. In each iteration, a new weak learner is inserted in order to reduce the total training error (Eq. (13)). 
Total_error $=\sum_{i} E\left[P_{t-1}\left(x_{i}\right)+a_{t} f\left(x_{i}\right)\right]$,

where $P_{t-1}\left(x_{i}\right)$ and $P^{\prime}(x)=a_{t} f\left(x_{i}\right)$ are the previous learner and the weak learner, respectively.

The main purpose of the Gradient Boosting of Regression Trees (GBRT) approach is to reduce the model's loss at each step by employing a gradient descent-like technique while adding weak learners. The decision tree algorithm is selected as the weak learner. The size of the decision tree algorithm is fixed. The model can be expressed as (Eq. (14)):

$$
\begin{aligned}
& F^{*}(x)=F_{m-1}^{*}(x)+\arg \min \sum_{i=1} L\left[y_{i}, F_{m-1}^{*}(x)+h(x)\right], \\
& g_{m}=-\frac{\partial L\left[y, F_{m-1}^{*}(x)\right]}{\partial F_{m-1}^{*}(x)},
\end{aligned}
$$

where $F_{m-1}{ }^{*}(x)$ is an imperfect model in the previous step, $h(x)$ is base regression trees, $g_{m}$ is the negative gradient of the loss function.

One of the most popular machine learning methods is XGBoost. It can be utilized for problems like regression and classification that need supervised learning. It is based on the gradient boosting architecture and is intended to broaden the scope of machine computation to produce a robust, portable, and precise library. The main distinction between XGBoost and GBRT is the structure of the objective function, which may be represented as (Eq. (15)):

$\operatorname{obj}(\theta)=\boldsymbol{L}(\theta)+\Omega(\theta)$,

where $\boldsymbol{L}(\theta)$ is the loss function, and $\boldsymbol{\Omega}(\theta)$ is the regularization term.

Zhang et al. [23] also developed a combination of support vector regression (SVR) and a genetic algorithm (GA) to approximate the deep beams' shear strength. The goal of merging the GA was to perform independent determination of the ideal parameter values and increase the efficiency of the SVR-GA model. The results of the research work of Feng et al [9], Zhang et al. [23], and proposed PSO-ANN are compared to the proposed hybrid technique. Table 5 shows the values of various machine learning methods. According to the results shown in Table 5, the ANN and RF models had the worst performance in predicting in terms of $R^{2}$, respectively. The AdaBoost, XGBoost, and GBRT models outperform the ANN and RF models. However, the hybrid models, PSO-ANN, SSA-ANN and the SVR-GA with a $R^{2}$ value of 0.95 , outperform other models.
Table $5 R^{2}$ values of various machine learning method

\begin{tabular}{lc}
\hline Models & $R^{2}$ \\
\hline PSO-ANN & 0.955 \\
SSA-ANN & 0.955 \\
AOA-ANN & 0.949 \\
RF [9] & 0.906 \\
AdaBoost [9] & 0.919 \\
GBRT [9] & 0.910 \\
XGBoost [9] & 0.928 \\
SVR-GA [23] & 0.958 \\
ANN [23] & 0.884
\end{tabular}

\subsection{Identifying important factors}

To provide black-box descriptions of the prediction model, researchers devised a number of ways. LIME [43] and its variations [44] rank among the most commonly cited. By adding variations to the feature vector parameters, these methods generate surrogate models for each predicted sample, learning the behavior of the reference model in the specific case of interest. The SHapley Additive exPlanations (SHAP) [45] take an alternative method, which is based on game theory. The feature relevance is determined using an approximation of Shapley values. In short, the weighted mean of the difference in prediction $f\left(S \cup f_{i}\right)-f(S)$ is used to evaluate the relevance of a feature $\left(f_{i}\right)$, where $S$ is a subset of the source set of features, $f$ is the original prediction model to be explained, and the data of the full set are considered missing. Fig. 11 shows the impact of each feature regarding the model output (PSO-ANN) using the overall SHAP values. The input parameters are listed on the $y$-axis in order of relevance (from top to bottom). It is seen from Fig. 10 that beam span $\left(l_{0}\right)$ has the highest positive impact in predicting shear strength, while $l_{0} / h$ has the highest negative impact in predicting shear strength. The beam width (b) and concrete compressive strength $\left(f_{c}\right)$ have a positive impact and takes third and fourth places in the order of importance for predicting shear strength, respectively. The strength of horizon reinforcement $\left(f_{y, h}\right)$ is one prominent variable with positive impact, while it appears a bit later (seven place). $a / h_{0}$ and $a$ take fifth and sixth places, negatively predicting the shear strength. Interestingly, the longitudinal reinforcement $(\rho)$ takes tenth place, but web reinforcement ratio in the vertical and horizontal direction $\left(\rho_{v, h}\right)$ take fourteenth and sixteenth places. 


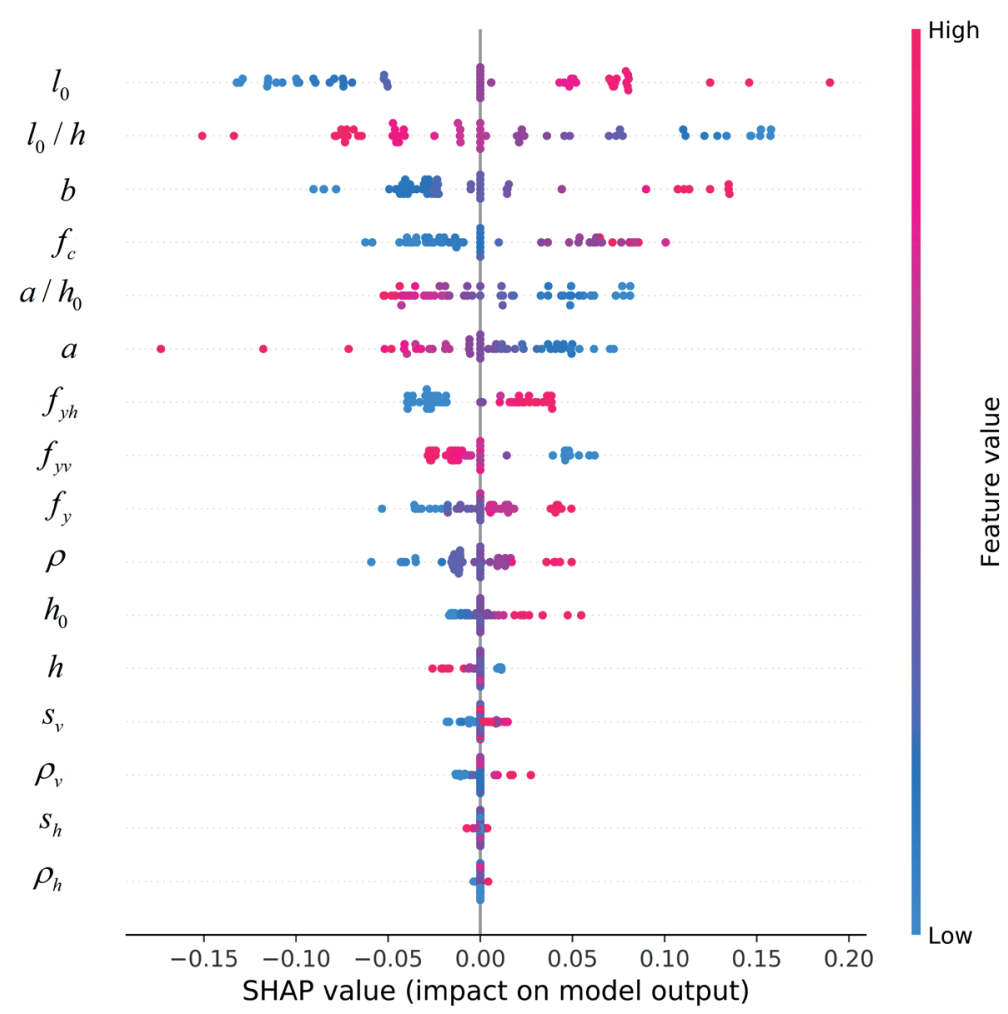

Fig. 11 Explanations for the hybrid model using SHAP method

\subsection{Comparisons with national design codes models}

For estimating the shear strength of RCD beams, national design codes offer empirical/mechanics-driven models. Here, design codes of China, US, Canada and Europe are considered for comparison with hybrid model (PSO-ANN). China's code formulation is a semi-empirical semi-analytical formula, whereas the other equations are developed using the strut-and-tie model. The following are the detailed expressions of Chinese code equation (Eq. (16), US code (Eq. (17)), Canadian code equation (Eq. (18)), and European code equation (Eq. (19) [46-49].

$$
\begin{aligned}
& V_{u}=\frac{1.75}{\lambda+1} f_{t} b h_{0}+\frac{l_{0} / h-2}{3} f_{y v} \frac{A_{s v}}{S_{h}} h_{0}+ \\
& \frac{5-l_{0} / h}{6} f_{y h} \frac{A_{s h}}{S_{v}} h_{0}, \lambda=a / h_{0},
\end{aligned}
$$

$V_{u}=0.85 \beta_{s} f_{c}^{\prime} b w_{s} \sin \theta, \theta=\arctan g\left(\frac{d_{b}}{a}\right) \geq 25^{\circ}$,

$w_{s}=\left[1.8 w_{t} \cos \theta+\left(l_{p E}+l_{p P}\right) \sin \theta\right] / 2$,

$V_{u}=\frac{f_{c}^{\prime}}{0.8+17 \varepsilon_{1}} b w_{s} \sin \theta$,

$\varepsilon_{1}=\varepsilon_{s}+\left(\varepsilon_{s}+0.002\right) \cot ^{2} \theta$,

$w_{s}=\frac{\left[1.88 w_{t} \cos \theta+\left(l_{p E}+l_{p P}\right) \sin \theta\right]}{2}$, where $f_{t}$ is the concrete tensile strength, $\beta_{s}$ is strut coefficient, $\theta$ is the angel between the strut and horizontal direction, $w_{s}$ is the width of the strut, $l_{p E}$ is the width of the top loading, $w_{t}$ is the height of the nodal region, $l_{p P}$ is the width of the bottom supporting plates, $\varepsilon_{s}$ is the tie's tensile strain, and the distance between the top and bottom nodal regions is denoted by $d_{b}$. The rest of the parameters are defined previously (Section 2). Table 6 includes statistical data on the distribution of predicted-to-test ratios (PTR) calculated using various methodologies. The mean, standard deviation (STD), and coefficient of variation (C.O.V.) value of PTR for the PSO-ANN are 1.00, 0.11, and 11.0\%, respectively. The hybrid model surpasses all four mechanical models.

Table 6 Performance comparisons between hybrid model and design

\begin{tabular}{|c|c|c|c|c|c|}
\hline \multirow{2}{*}{ Models } & \multicolumn{5}{|c|}{ The ratio of predicted/tested value } \\
\hline & Min. & Max. & Mean & STD & C.O.V. $(\%)$ \\
\hline $\begin{array}{l}\text { Chinese code: } \\
\text { GB50010-2010 }\end{array}$ & 0.36 & 3.24 & 1.43 & 0.39 & 27.0 \\
\hline US code: ACI 318 & 0.30 & 5.27 & 1.57 & 0.69 & 43.9 \\
\hline $\begin{array}{l}\text { Canadian code: CSA } \\
\text { A23.3-04 }\end{array}$ & 0.59 & 4.50 & 1.56 & 0.56 & 35.8 \\
\hline European code: EU2 & 0.44 & 3.47 & 1.42 & 0.54 & 38.0 \\
\hline PSO-ANN & 0.76 & 1.40 & 1.0 & 0.11 & 11.0 \\
\hline
\end{tabular}
codes models 


\section{Conclusions}

The estimation of the shear capacity of reinforced concrete deep (RCD) beams has become a difficult and important task that has attracted the interest of researchers. This research looks into the possibility of employing a hybrid model to predict the shear capacity of RCD beams. To construct the comprehensive relationship between the shear strength capacity and its affecting components, three metaheuristic algorithms, including particle swarm optimization (PSO), Archimedes optimization algorithm (AOA), sparrow search algorithm (SSA), are combined with the artificial neural network (ANN). The creation and validation of the models were carried out using a data set gathered using prior studies, which included 271 test results. The results show that the PSO-ANN, AOA_ANA, and SSA-ANN models perform well with a coefficient of determination of $0.955,0.949$, and 0.955 , respectively. Among the hybrid models, the PSO-ANN model was more accurate, by using fewer neurons in the hidden layer. Six well-established machine learning predictive models, which were utilized by other researchers, were considered in this study for comparison and validation, including RF, AdaBoost, GBRT, XGBoost, SVR-GA, and ANN. These results suggest that the hybrid models (PSO-ANN and SVR-GA) have acceptable performance and outperform other machine learning predictive models. The application of the SHapley Additive exPlanations approach to rank input parameters and describe the contributing elements to the shear capacity of the RCD beams is further explored in this paper. It should be noted that beam span $\left(l_{0}\right)$ has the highest positive impact in predicting shear strength, while

\section{References}

[1] Bao, Y., Tang, Z., Li, H. "Compressive-sensing data reconstruction for structural health monitoring: a machine-learning approach", Structural Health Monitoring, 19(1), pp. 293-304, 2020. https://doi.org/10.1177\%2F1475921719844039

[2] Gardner, P., Fuentes, R., Dervilis, N., Mineo, C., Pierce, S. G., Cross, E. J., Worden, K. "Machine learning at the interface of structural health monitoring and non-destructive evaluation", Philosophical Transactions of the Royal Society A, 378(2182), Article number: 20190581, 2020.

https://doi.org/10.1098/rsta.2019.0581

[3] Torky, A. A., Ohno, S. "Deep learning techniques for predicting nonlinear multi-component seismic responses of structural buildings", Computers \& Structures, 252, Article number: 106570, 2021. https://doi.org/10.1016/j.compstruc.2021.106570

[4] Zheng, Y., Xie, Y., Long, X. "A comprehensive review of Bayesian statistics in natural hazards engineering", Natural Hazards, 108, pp. 63-91, 2021.

https://doi.org/10.1007/s11069-021-04729-2 $l_{0} / h$ has the highest negative impact in predicting shear strength. The results of this study demonstrate the significant potential of the hybrid model for predicting predict the shear capacity of the RCD beams. The model can be used in a variety of ways in structural design and analysis. For example, in the design stage of the RCD beams, it can be used to quickly anticipate shear strength and examine the impact of various design factors. It can also be used as a generic tool for evaluating the performance of various mechanical models and empirical formulas in design standards. However, there is still potential for development in this field, such as applying active learning to enhance computing efficiency, establishing a mechanics-guided shear strength framework based on machine learning for better application, and so on. To better anticipate the shear strength of the RCD beams, a larger dataset range also must be developed in the future.

\section{Declaration of interests}

The authors declare that they have no known competing financial interests or personal relationships that could have appeared to influence the work reported in this paper.

\section{Funding}

This research did not receive any specific grant from funding agencies in the public, commercial, or not-for-profit sectors.

\section{Data availability}

The data that support the findings of this study are available from the corresponding author upon reasonable request.

[5] Ghorbani, S., Barari, M., Hosseini, M. "A modern method to improve of detecting and categorizing mechanism for micro seismic events data using boost learning system", Civil Engineering Journal, 3(9), pp. 715-726, 2017. https://doi.org/10.21859/cej-03098

[6] Barkhordari, M., Es-haghi, M. S., "Straightforward Prediction for Responses of the Concrete Shear Wall Buildings Subject to Ground Motions Using Machine Learning Algorithms", International Journal of Engineering, 34(7), pp. 1586-1601, 2021.

https://doi.org/10.5829/IJE.2021.34.07A.04

[7] Barkhordari, M., Tehranizadeh, M. "Ranking passive seismic control systems by their effectiveness in reducing responses of highRise buildings with concrete shear walls using multiple-Criteria decision making", International Journal of Engineering, 33(8), pp. 1479-1490, 2020. https://doi.org/10.5829/ije.2020.33.08b.06 
[8] Nguyen, H. D., Truong, G. T., Shin, M. "Development of extreme gradient boosting model for prediction of punching shear resistance of r/c interior slabs", Engineering Structures, 235, Article number: 112067, 2021. https://doi.org/10.1016/j.engstruct.2021.112067

[9] Feng, D.-C., Wang, W.-J., Mangalathu, S., Hu, G., Wu, T. "Implementing ensemble learning methods to predict the shear strength of RC deep beams with/without web reinforcements", Engineering Structures, 235, Article number: 111979, 2021. https://doi.org/10.1016/j.engstruct.2021.111979

[10] Moradi, M. J., Roshani, M. M., Shabani, A., Kioumarsi, M. "Prediction of the load-bearing behavior of SPSW with rectangular opening by RBF network", Applied Sciences, 10(3), Article number: $1185,2020$.

https://doi.org/10.3390/app10031185

[11] Barkhordari, M. S.,Tehranizadeh, M, "Response estimation of reinforced concrete shear walls using artificial neural network and simulated annealing algorithm", Structures, 34, pp. 1155-1168, 2021. https://doi.org/10.1016/j.istruc.2021.08.053

[12] Jueyendah, S., Lezgy-Nazargah, M., Eskandari-Naddaf, H., Emamian, S. A. "Predicting the mechanical properties of cement mortar using the support vector machine approach", Construction and Building Materials, 291, Article number: 123396, 2021. https://doi.org/10.1016/j.conbuildmat.2021.123396

[13] Park, J., Kuchma, D. "Strut-and-tie model analysis for strength prediction of deep beams", ACI Structural Journal, 104(6), pp. 657666, 2007.

[14] Lezgy-Nazargah, M., Dezhangah, M., Sepehrinia, M. "The effects of different FRP/concrete bond-slip laws on the 3D nonlinear FE modeling of retrofitted RC beams-A sensitivity analysis", Steel and Composite Structures, 26(3), pp. 347-360, 2018. https://doi.org/10.12989/scs.2018.26.3.347

[15] Tang, C. Y., Tan, K. H. "Interactive mechanical model for shear strength of deep beams", Journal of Structural Engineering, 130(10), pp. 1534-1544, 2004. https://doi.org/10.1061/(ASCE)0733-9445(2004)130:10(1534)

[16] Lezgy-Nazargah, M. "A four-variable global-local shear deformation theory for the analysis of deep curved laminated composite beams", Acta Mechanica, 231(4), pp. 1403-1434, 2020. https://doi.org/10.1007/s00707-019-02593-7

[17] Lezgy-Nazargah, M. "An efficient materially nonlinear finite element model for reinforced concrete beams based on layered global-local kinematics", Acta Mechanica, 229(3), pp. 1429-1449, 2018. https://doi.org/10.1007/s00707-017-2081-3

[18] ACI Committee 318 "Building Code Requirements for Structural Concrete (ACI 318-19) - Commentary on Building Code Requirements for Structural Concrete (ACI 318R-19)", American Concrete Institute, Farmington Hills, MI, USA, 2019.

[19] CSA Group "A23.3-14 Design of concrete structures", CSA Group, Toronto, Canada, 2019.

[20] Fu, B., Feng, D.-C. "A machine learning-based time-dependent shear strength model for corroded reinforced concrete beams", Journal of Building Engineering, 36, Article number: 102118, 2021. https://doi.org/10.1016/j.jobe.2020.102118
[21] Al-Musawi, A. A., Alwanas, A. A. H., Salih, S. Q., Ali, Z. H., Tran, M. T., Yaseen, Z. M. "Shear strength of SFRCB without stirrups simulation: implementation of hybrid artificial intelligence model", Engineering with Computers, 36(1), pp. 1-11, 2020. https://doi.org/10.1007/s00366-018-0681-8

[22] Sharafati, A., Haghbin, M., Aldlemy, M. S., Mussa, M. H., Al Zand, A. W., Ali, M., Bhagat, S. K., Al-Ansari, N., Yaseen, Z. M. "Development of advanced computer aid model for shear strength of concrete slender beam prediction", Applied Sciences, 10(11), Article number: 3811, 2020. https://doi.org/10.3390/app10113811

[23] Zhang, G., Ali, Z. H., Aldlemy, M. S., Mussa, M. H., Salih, S. Q., Hameed, M. M., Al-Khafaji, Z. S., Yaseen, Z. M. "Reinforced concrete deep beam shear strength capacity modelling using an integrative bio-inspired algorithm with an artificial intelligence model", Engineering with Computers, 2020. https://doi.org/10.1007/s00366-020-01137-1

[24] Chou, J.-S., Ngo, N.-T., Pham, A.-D. "Shear strength prediction in reinforced concrete deep beams using nature-inspired metaheuristic support vector regression", Journal of Computing in Civil Engineering, 30(1), Article number: 04015002, 2016. https://doi.org/10.1061/(ASCE)CP.1943-5487.0000466

[25] Shahbazian, A., Rabiefar, H., Aminnejad, B. "Shear Strength Determination in RC Beams Using ANN Trained with Tabu Search Training Algorithm", Advances in Civil Engineering, 2021, Article ID 1639214, 2021. https://doi.org/10.1155/2021/1639214

[26] Nikoo, M., Aminnejad, B., Lork, A. "Predicting Shear Strength in FRP-Reinforced Concrete Beams Using Bat Algorithm-Based Artificial Neural Network", Advances in Materials Science and Engineering, 2021, Article ID 5899356, 2021. https://doi.org/10.1155/2021/5899356

[27] Nguyen, T.-A., Ly, H.-B., Mai, H.-V. T., Tran, V. Q. "On the Training Algorithms for Artificial Neural Network in Predicting the Shear Strength of Deep Beams", Complexity, 2021, Article ID 5548988, 2021 https://doi.org/10.1155/2021/5548988

[28] Kleinberg, B., Li, Y., Yuan, Y. "An alternative view: When does SGD escape local minima?", In: Proceedings of the 35th International Conference on Machine Learning, Stockholm, Sweden, 2018, pp. 2688-2697.

[29] Sun, R., Li, D., Liang, S., Ding, T., Srikant, R. "The global landscape of neural networks: An overview", IEEE Signal Processing Magazine, 37(5), pp. 95-108, 2020. https://doi.org/10.1109/MSP.2020.3004124

[30] Cheng, M.-Y., Cao, M.-T. "Evolutionary multivariate adaptive regression splines for estimating shear strength in reinforced-concrete deep beams", Engineering Applications of Artificial Intelligence, 28, pp. 86-96, 2014. https://doi.org/10.1016/j.engappai.2013.11.001

[31] Asteris, P. G., Armaghani, D. J., Hatzigeorgiou, G. D., Karayannis, C. G., Pilakoutas, K. "Predicting the shear strength of reinforced concrete beams using Artificial Neural Networks", Computers and Concrete, 24(5), pp. 469-488, 2019. https://doi.org/10.12989/cac.2019.24.5.469 
[32] Mohammadhassani, M., Nezamabadi-Pour, H., Jumaat, M., Jameel, M., Hakim, S. J. S., Zargar, M., "Application of the ANFIS model in deflection prediction of concrete deep beam", Structural Engineering and Mechanics, 45(3), pp. 323-336, 2013.

[33] Swingler, K. "Applying neural networks: a practical guide", Morgan Kaufmann, San Francisco, CA, USA, 1996.

[34] Hornik, K., Stinchcombe, M., White, H. "Multilayer feedforward networks are universal approximators", Neural Networks, 2(5), pp. 359-366, 1989. https://doi.org/10.1016/0893-6080(89)90020-8

[35] Lu, Y., Liang, M., Ye, Z., Cao, L. "Improved particle swarm optimization algorithm and its application in text feature selection", Applied Soft Computing, 35, pp. 629-636, 2015. https://doi.org/10.1016/j.asoc.2015.07.005

[36] Clerc, M., Kennedy, J. "The particle swarm-explosion, stability, and convergence in a multidimensional complex space", IEEE Transactions on Evolutionary Computation, 6(1), pp. 58-73, 2002. https://doi.org/10.1109/4235.985692

[37] Hashim, F. A., Hussain, K., Houssein, E. H., Mabrouk, M. S., Al-Atabany, W. "Archimedes optimization algorithm: a new metaheuristic algorithm for solving optimization problems", Applied Intelligence, 51(3), pp. 1531-1551, 2021. https://doi.org/10.1007/s10489-020-01893-Z

[38] Xue, J., Shen, B. "A novel swarm intelligence optimization approach: sparrow search algorithm", Systems Science \& Control Engineering, 8(1), pp. 22-34, 2020. https://doi.org/10.1080/21642583.2019.1708830

[39] Masters, T. "Practical neural network recipes in C++", Academic Press, San Diego, CA, USA, 1993. https://doi.org/10.1016/C2009-0-22399-3

[40] Stone, M. "Cross-validatory choice and assessment of statistical predictions", Journal of the Royal Statistical Society: Series B (Methodological), 36(2), pp. 111-133, 1974. [online] Available at: https://www.jstor.org/stable/2984809
[41] Kuhn, M., Johnson, K. "Applied Predictive Modeling", Springer, New York, NY, USA, 2013. https://doi.org/10.1007/978-1-4614-6849-3

[42] Slowik, A. "Swarm intelligence algorithms: modifications and applications", CRC Press, Boca Raton, FL, USA, 2020.

[43] Ribeiro, M. T., Singh, S., Guestrin, C. "Why should i trust you? Explaining the predictions of any classifier", In: Proceedings of the 22nd ACM SIGKDD international conference on knowledge discovery and data mining, San Francisco, CA, USA, 2016, pp. $1135-1144$.

https://doi.org/10.1145/2939672.2939778

[44] Ribeiro, M. T., Singh, S., Guestrin, C. "Anchors: High-precision model-agnostic explanations", In: Proceedings of the AAAI Conference on Artificial Intelligence, 32(1), New Orleans, LA, USA, 2018, pp. $1527-1535$.

[45] Lundberg, S. M., Lee, S.-I. "A unified approach to interpreting model predictions", In: Proceedings of the 31 st international conference on neural information processing systems, Long Beach, CA, USA, 2017, pp. 4768-4777.

[46] Chinese Standard "GB 50010 - 2002 Code for design of concrete structures", National Standard of the People's Republic of China, Beijing, China, 2002.

[47] ACI Committee 318 "ACI CODE-318-19: Building code requirements for structural concrete", American Concrete Institute, Farmington Hills, MI, USA, 2014.

[48] CSA "A23.3-04 Design of concrete structures", Canadian Standards Association, Mississauga, ON, Canada, 2004.

[49] Hendy, C. R., Smith, D. A. "Designers' Guide to EN 1992-2: Eurocode 2: Design of Concrete Structures: Part 2: Concrete bridges", Thomas Telford, London, UK, 2007. 